(C) 2007 JEMWA . Reprinted with permission from:

Abdulla A. Abouda, H.M. El-Sallabi, Lasse Vuokko and S.G. Häggman, "Spatial Smoothing Effect on Kronecker MIMO Channel Model in Urban Microcells," Journal of Electromagnetic Waves and Applications JEMWA, vol. 21, no. 5, pp. 681-696, 2007. 


\title{
SPATIAL SMOOTHING EFFECT ON KRONECKER MIMO CHANNEL MODEL IN URBAN MICROCELLS
}

\author{
A. A. Abouda, H. M. El-Sallabi, L. Vuokko \\ and S. G. Häggman
}

Helsinki University of Technology

P.O. Box 3000, FIN-02015 HUT, Finland

\begin{abstract}
In this paper, the effect of spatial smoothing on performance of widely used stochastic narrowband multiple-input multiple-output (MIMO) radio channel model, namely the Kronecker model, is investigated based on data measured in urban microcellular environment at $5.3 \mathrm{GHz}$ carrier frequency. Results from non-line of sight (NLOS) and line of sight (LOS) traveling routes are analyzed. It is noticed that in NLOS traveling route spatial smoothing improves the accuracy of the large eigenvalues of the channel correlation matrix and significantly enhances the applicability of the Kronecker structure. Under LOS condition both the Kronecker model and its smoothed version fail to render the eigenvalues of the measured channel correlation matrix but spatial smoothing slightly improves the applicability of the Kronecker structure.
\end{abstract}

\section{INTRODUCTION}

A number of channel models have been developed for characterizing multiple-input multiple-output (MIMO) wireless channels, e.g., [1-3]. Based on channel models several studies have been carried out for deep understanding of MIMO systems performance, e.g., [4]. The Kronecker stochastic MIMO radio channel model is one of these models that has been extensively used. However, it has been widely noticed that this channel model has shown different performances under different propagation scenarios. While in $[2,3]$ it is shown that the Kronecker model is very suitable for matching results from measured data with small number of transmit and receive antenna elements, in other studies $[5,6]$ significant differences between the results from the measured data and the predicted results are reported when large number of transmit and receive antennas is used. Although, 
the simplicity of the Kronecker model makes it an attractive starting point in the analysis of any space-time processing technique.

In this paper, the effect of spatial smoothing on the performance of the Kronecker model is investigated based on data measured in urban microcellular environment at $5.3 \mathrm{GHz}$ carrier frequency. Results from non line-of-sight (NLOS) and line-of-sight (LOS) traveling routes are analyzed. The investigation is performed by comparing the measured channel transfer matrices to the channel transfer matrices obtained from the Kronecker model and its spatially smoothed version in terms of the distribution of the channel coefficients and the distribution of the eigenvalues of the channel correlation matrix. The distributions of the measured and modeled quantities are compared by utilizing the Kolmogorov-Smirnov (KS) goodness test. Furthermore, the channel capacity and the symbol error rate (SER) performance achievable over the measured and modeled channels are compared. The validity of the Kronecker structure in modeling the full channel correlation matrix is also assessed by analyzing the model error performance measure.

\section{SYSTEM MODEL AND SIGNALING SCHEME}

Consider a narrowband MIMO wireless communication system with $N_{t}$ transmit antennas and $N_{r}$ receive antennas. The system employs spatial multiplexing scheme where different signals are transmitted from each transmit antenna simultaneously. Under this signaling scheme and an uniform power allocation strategy the input-output relation between the transmitted and received signals can be written as:

$$
\mathbf{y}=\mathbf{H x}+\mathbf{n}
$$

where $\mathbf{y} \in C^{N_{r}, 1}$ is the received signal vector, $\mathbf{x} \in C^{N_{t}, 1}$ is the transmitted signal vector with covariance matrix $R_{x}=E\left\{\mathbf{x x}^{H}\right\}=$

$\frac{\sigma_{x}^{2}}{N_{t}} \mathbf{I}_{N_{t}}$, where $(.)^{H}$ denotes Hermitian transposition, $\sigma_{x}^{2}$ is the total transmitted signal power and $\mathbf{I}_{N}$ denotes identity matrix of size $N \times N$, $\mathbf{H} \in C^{N_{r}, N_{t}}$ is a narrowband normalized channel matrix and $\mathbf{n} \in C^{N_{r}, 1}$ is zero mean complex Gaussian receiver noise vector with covariance matrix $E\left\{\mathbf{n n}^{H}\right\}=N_{o} \mathbf{I}_{N_{r}}$.

\section{KRONECKER MODEL STRUCTURE}

The main idea of the Kronecker stochastic MIMO radio channel model is to generate a correlated fading channel matrix using an independent identical distributed (iid) complex Gaussian matrix and transmitter and receiver spatial correlation information. The Kronecker model was 
developed to account for spatial correlation between antenna elements in a realistic way under the assumption that the spatial correlation at the transmitter and receiver ends are independent $[2,3]$.

Utilizing the Kronecker model, a channel matrix $\mathbf{H}_{k r o n}$ can be generated according to:

$$
\mathbf{H}_{k r o n}=\mathbf{R}_{r x}^{1 / 2} \mathbf{H}_{i i d}\left(\mathbf{R}_{t x}^{1 / 2}\right)^{T}
$$

where $\mathbf{H}_{i i d} \in C^{N_{r}, N_{t}}$ is a random matrix with iid zero mean and unit variance complex Gaussian elements, $(.)^{T}$ denotes transpose operation, $\mathbf{A}^{1 / 2}$ denotes square root of matrix $\mathbf{A}$, and $\mathbf{R}_{t x}$ and $\mathbf{R}_{r x}$ are the transmitter and receiver spatial correlation matrices, respectively. The two ends correlation matrices can be obtained either from measured data or theoretical studies. Using measurement data, these correlation matrices can be extracted as follows $[2,3]$ :

$$
\begin{aligned}
& \mathbf{R}_{t x}=E\left\{\left(\mathbf{H}_{\text {meas }}^{H} \mathbf{H}_{\text {meas }}\right)^{T}\right\} \\
& \mathbf{R}_{r x}=E\left\{\mathbf{H}_{\text {meas }} \mathbf{H}_{\text {meas }}^{H}\right\}
\end{aligned}
$$

where $\mathbf{H}_{\text {meas }} \in C^{N_{r}, N_{t}}$ is the narrowband normalized measured channel matrix and $E\{$.$\} denotes expectation operator that averages$ over all measured channel matrices. It is shown in [2][3] that the generated channel matrix, $\mathbf{H}_{k r o n}$, exhibits statistical properties similar to the measured channel matrix. Furthermore, it is also shown that the full channel correlation matrix can be written as the Kronecker product of the correlation matrices seen by both ends as:

$$
\mathbf{R}_{k r o n}=\mathbf{R}_{t x} \otimes \mathbf{R}_{r x}
$$

where $\otimes$ denotes the Kronecker product. It should be noticed that the full channel correlation matrix can be obtained from the measured data as:

$$
\mathbf{R}=E\left\{\operatorname{vec}\left(\mathbf{H}_{\text {meas }}\right) \operatorname{vec}\left(\mathbf{H}_{\text {meas }}\right)^{H}\right\}
$$

where $v e c(\mathbf{A})$ denotes vector operator that stacks the matrix $\mathbf{A}$ into a vector columnwise.

\section{MOTIVATIONS FOR CONSIDERING SPATIAL SMOOTHING}

The independency between the transmitter and receiver spatial correlation matrices and the wide sense stationarity (WSS) are two corner stone assumptions required for the applicability of the Kronecker model. The former assumption necessities that calculating 
the spatial correlation matrix of one end is independent on the selected antenna element in the other end. The independency assumption is expected to be fulfilled when the angles of departure in one end are independent on the angles of arrival in the other end. This is the case when there is no strong component connecting the two communication ends as the case of pure NLOS scenario. On the other side, the WSS assumption requires that the spatial correlation properties at both ends do not change significantly over the traveling route. General speaking, this assumption can be considered fulfilled in indoor environment where the mobile terminal moves relatively short distances, e.g., (1-3 m), and the propagation environment does not change significantly over traveling route. When the correlation properties change significantly over the traveling route, averaging over the whole route may result in loss in valuable spatial correlation information and consequently in inaccurate MIMO channel modeling.

Spatial smoothing technique is used to resolve correlated sources. It has been used to improve the performance of angle of arrival estimation algorithms in presence of correlated sources [7]. Our study of the effect of spatial smoothing on the performance of the Kronecker model is motivated by the validity of the independency and WSS assumptions above. Spatial smoothing of the transmitter and receiver correlation matrices will have decorrelation effect on the two ends spatial correlation matrices in one hand and will divide the whole traveling route to small sub segments which in turn improves the validity of the WSS assumption.

With spatial smoothing the structure of the Kronecker model remains same as in (2) and the transmitter and receiver spatial correlation matrices are redefined as:

$$
\begin{aligned}
\hat{\mathbf{R}}_{t x} & =E_{s}\left\{\left(\mathbf{H}_{\text {meas }}^{H} \mathbf{H}_{\text {meas }}\right)^{T}\right\} \\
\hat{\mathbf{R}}_{r x} & =E_{s}\left\{\mathbf{H}_{\text {meas }} \mathbf{H}_{\text {meas }}^{H}\right\}
\end{aligned}
$$

where $E_{s}\{$.$\} denotes spatial smoothing defined as E_{s}\{x(n)\}=$ $\frac{1}{w} \sum_{i=n}^{n+w} x(i)$ where $w$ is the window width. Spatial smoothing will improve the tractability of the Kronecker model to the changes in the spatial correlation of the measured data. However, this improvement comes as a cost of more complexity of the model since a large number of correlation matrices should be stored compared to one channel correlation matrix for each end in the Kronecker model. It should be noticed that the aim of this study is not to introduce modified Kronecker model rather than to gain deep understating of the performance of the Kronecker model in urban microcells. 


\section{MEASUREMENT CAMPAIGN AND ENVIRONMENT DESCRIPTION}

The measurement campaign was carried out at downtown of Helsinki at $5.3 \mathrm{GHz}$ carrier frequency. The measurement campaign represents an urban microcellular environment where a transmitter equipped with 16 elements dual-polarized planner antenna was located in the main street below the rooftops level at height of $10 \mathrm{~m}$. A pseudonoise code with $60 \mathrm{MHz}$ chip frequency was transmitted with power limited to $37 \mathrm{dBm}$. A receiver equipped with 15 directive and dual-polarized semispherical antenna at height of $1.6 \mathrm{~m}$ was moved in different streets to create different routes. The receiver velocity was approximately $0.2 \mathrm{~m} / \mathrm{s}$, meaning that during the measurement of one complex channel matrix the receiver was moved a distance of $0.014 \mathrm{~m}$.

The results presented in this study are based on subset of data taken from two measured traveling routes, NLOS and LOS. In the NLOS route the receiver terminal was moved in a street perpendicular to the main street with no LOS component while in the LOS route the receiver terminal was moved in the main street where direct LOS component between the transmitter and receiver terminal exist. The propagation environment is shopping area with common glass structure in the first floor. In each route an appropriate subset of channel matrices with 7 transmit and 7 receive antennas along measurement route of $750 \lambda$ is considered, where $\lambda$ is the wavelength.

\section{DATA ANALYSIS}

A successful channel model is expected to imitate similar statistical properties of the measured channel transfer matrices. Statistical properties such as the distribution of the channel coefficients and the distribution of the eigenvalues of the channel correlation matrix are expected to be accurately rendered by the channel mode. In addition, the achievable channel capacity and the SER performance over the measured channel are also expected to be accurately predicted. These are common performance measures used to validate the performance of a channel model based on measured data. In addition, there is another less common performance measure specific for the Kronecker structure that was used in [3] and [6]. The model error that measures how good the full channel correlation matrix can be modeled as the Kronecker product of the two ends correlation matrices. All these performance measures are described briefly in the following subsections followed by numerical results based on the measured data and intuitive discussions.

In the following analysis all the encountered channel matrices are 
normalized according to:

$$
\|\mathbf{H}\|_{F}^{2}=N_{r} \times N_{t}
$$

where $\|.\|_{F}$ denotes matrix Frobenius norm.

Kolmogorov-Smirnov (KS) test provides a metric to decide whether a given sample belongs to a specific distribution or not. The same metric can be also used to verify if two given random variables follow the same distribution or not. The KolmogorovSmirnov metric (KSM) measures the maximum difference between the empirical cumulative distribution functions (CDF) of two random variables $x_{1}$ and $x_{2}$ as [9]:

$$
K S M\left(x_{1}, x_{2}\right)=\max _{x}\left(\left|C D F\left(x_{1}\right)-C D F\left(x_{2}\right)\right|\right)
$$

The hypothesis regarding the distributional similarity is accepted or rejected according to the KSM at specific significance level. Since we are dealing with random variables, it is very helpful to utilize this metric in the following analysis.

\subsection{Channel Coefficients Distribution Analysis}

The measured envelopes and phases are compared to Rayleigh and uniform distributions, respectively, in order to gain intuitive sense of the propagation environment and assess the applicability of the Kronecker model in matching the measured data. One should be aware that studying the distribution of one element from the channel matrix may not give enough information about the propagation environment, therefore, the distributions of the all channel elements are considered.

\subsubsection{Envelopes of Channel Coefficients Distribution Analysis}

Figure 1 shows the KSM comparing the envelopes of the measured channel coefficients to Rayleigh distribution in NLOS and LOS traveling routes. One can observe that the different measured envelopes show different degree of similarity to the Rayleigh distribution. In NLOS route $32.65 \%$ of the measured envelopes pass the $\mathrm{KS}$ test at $95 \%$ significance level. It is worthy to notice that at $95 \%$ significance level the decision threshold is about 0.0248. Channel coefficients with KSM large than this threshold fail to pass the test. However, the maximum differences between the CDFs of the measured envelopes and the CDF of the Rayleigh distribution in NLOS route are slightly small. They range from 0.013 in channel coefficient $H(7,5)$ to 0.122 in channel coefficient $H(3,4)$ with average of 0.056 , where 
$H(i, j)$ denotes one element from the channel matrix with ith row and $\mathrm{j} t h$ column. In LOS route the percentage of the measured envelopes pass the KS test is reduced to $8.16 \%$ at the same significance level. Channel coefficients did not pass the KS test with Rayleigh distribution reflect the presence of strong component which diverts the Rayleigh distribution to more Rice one.

The ability of the Kronecker model and its smoothed version in rendering the distribution of the measured envelopes is studied by analyzing the KSM for the envelopes. Figure 2 shows the KSM comparing the envelopes rendered by the Kronecker model and its smoothed version with $w=40 \lambda$ to the measured envelopes in NLOS traveling route. While the Kronecker model can match $12.25 \%$ of the measured envelopes at 95\% significance level, its smoothed version can render $14.28 \%$ of the measured envelopes at the same significant level.

In LOS route the effect of spatial smoothing on the capability of the Kronecker model to render the measured channel envelopes is significant as it can be seen from Figure 3, where the KSM comparing the envelopes rendered by the Kronecker model and its smoothed version with $w=40 \lambda$ to the LOS measured envelopes is shown. While the Kronecker model fails to render the measured envelopes at $95 \%$ significance level, its smoothed version reduces the differences between the measured and modeled envelopes significantly. With spatial smoothing the percentage of channel envelopes pass the KS test is about $10.2 \%$ of the envelopes at $95 \%$ significance level.
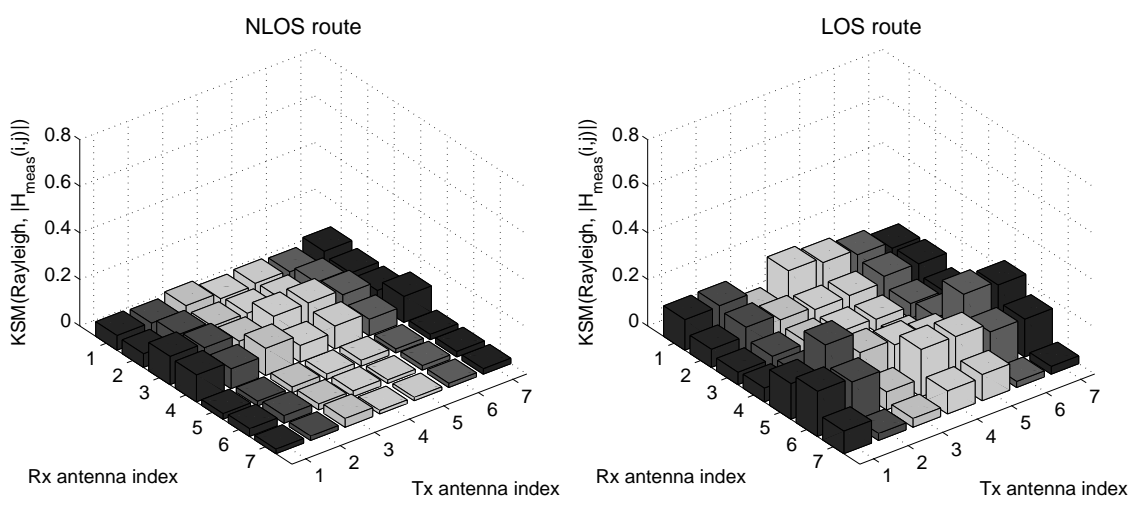

Figure 1. KSM comparing the envelopes of the measured channel coefficients in NLOS and LOS traveling routes to Rayleigh distribution. 

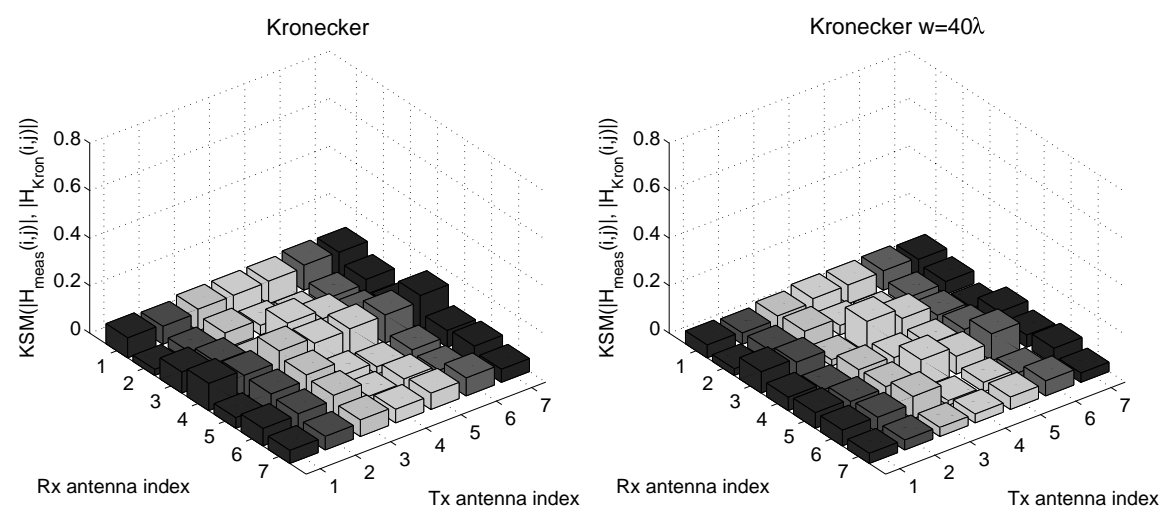

Figure 2. KSM comparing the envelopes of channel coefficients rendered by the Kronecker model and its smoothed version to the measured envelopes in NLOS traveling route.
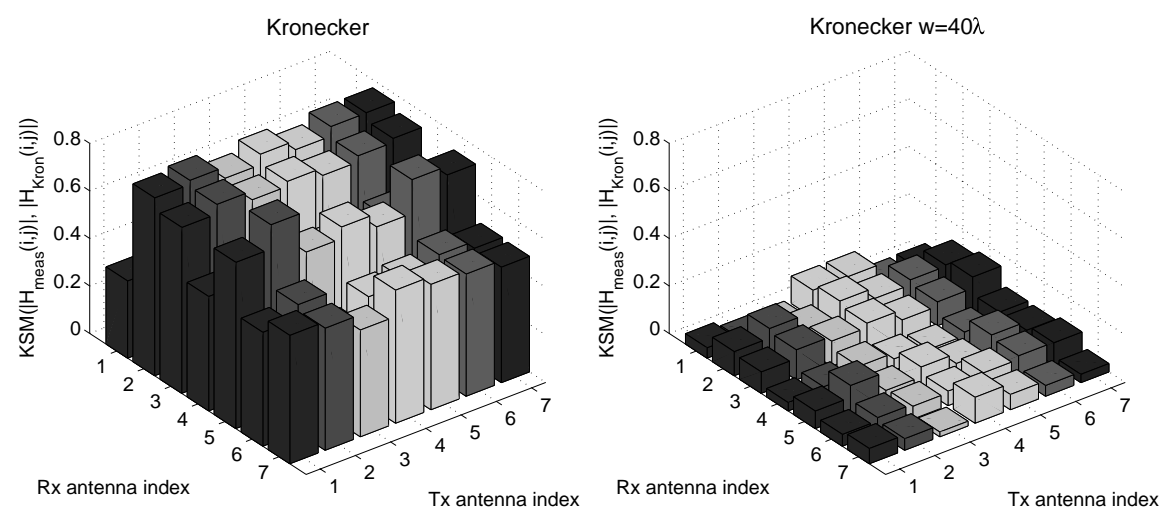

Figure 3. KSM comparing the envelopes of channel coefficients rendered by the Kronecker model and its smoothed version to the measured envelopes in LOS traveling route.

\subsubsection{Phases of Channel Coefficients Distribution Analysis}

In Figure 4 the phases of the measured channel coefficients are compared to uniformly distributed random variable varying from $-\pi$ to $\pi$ using the KSM. It is noticed that the phases of the measured channel coefficients in both NLOS and LOS routes follow the uniform distribution very well where the percentage of the phases of the channel coefficients pass the KS test at $95 \%$ significance level is $100 \%$. The phases distribution rendered by the Kronecker model and its smoothed 

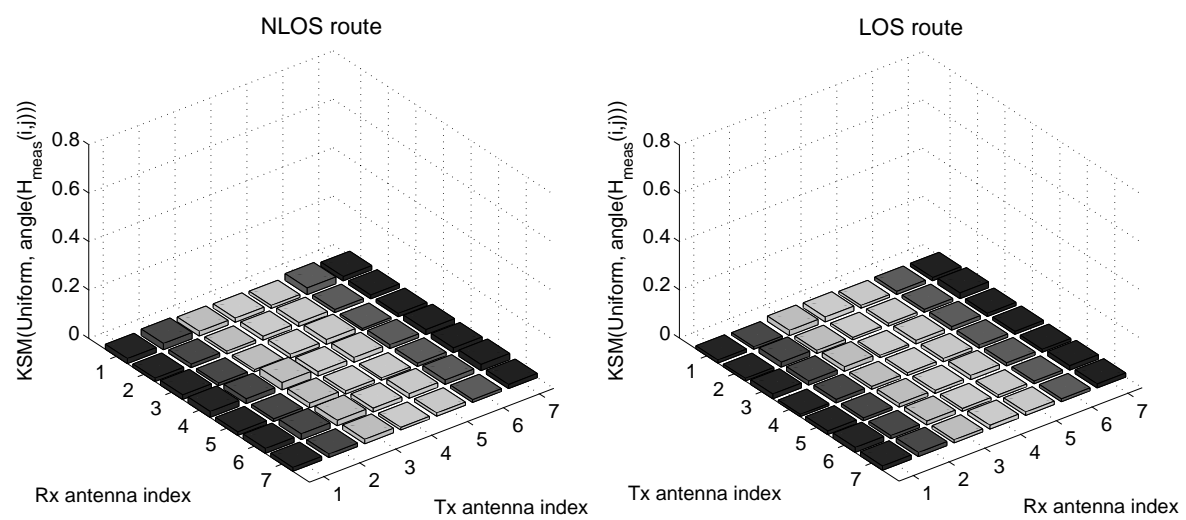

Figure 4. KSM comparing the phases of the measured channel coefficients in NLOS and LOS traveling routes to uniform distribution.

version in both NLOS and LOS traveling routes were found to follow the uniform distribution very well. In NLOS and LOS routes $91.83 \%$ and $87.75 \%$ of the phases of the channel coefficients obtained by the Kronecker model match the measured phases at $95 \%$ significance level, respectively. With spatial smoothing the percentage goes to $93.87 \%$ in NLOS route and $91.83 \%$ in LOS route at the same significance level.

\subsection{Eigenvalues Distribution Analysis}

The distribution of the eigenvalues of the channel correlation matrix reveals valuable information about the MIMO channel characteristics and consequently predicts the MIMO system performance. The eigenvalues are obtained by applying the eigenvalue decomposition on the channel correlation matrix as follows:

$$
\lambda_{i}=\operatorname{EVD}\left(\mathbf{H H}^{H}\right), \quad i=1,2, \ldots, R\left(\mathbf{H} \mathbf{H}^{H}\right)
$$

where $\lambda_{i}$ is the $i$ th eigenvalue of the channel correlation matrix, $\operatorname{EVD}(\mathbf{A})$ returns the eigenvalues of matrix $\mathbf{A}$ and $R(\mathbf{A})$ denotes the rank of matrix $\mathbf{A}$.

The distribution of the eigenvalues of the measured channel correlation matrices in addition to the eigenvalues of the iid channel are shown in Figure 5. Useful information about the propagation scenarios can be extracted from the eigenvalues plot. In terms of spatial multiplexing gain, one can observe that there are six and five spatial channels available $90 \%$ of the time in the NLOS and LOS traveling routes, respectively, since there are six and five non zero eigenvalues exist $90 \%$ of the time, (this is can be seen from the CDF graph at 


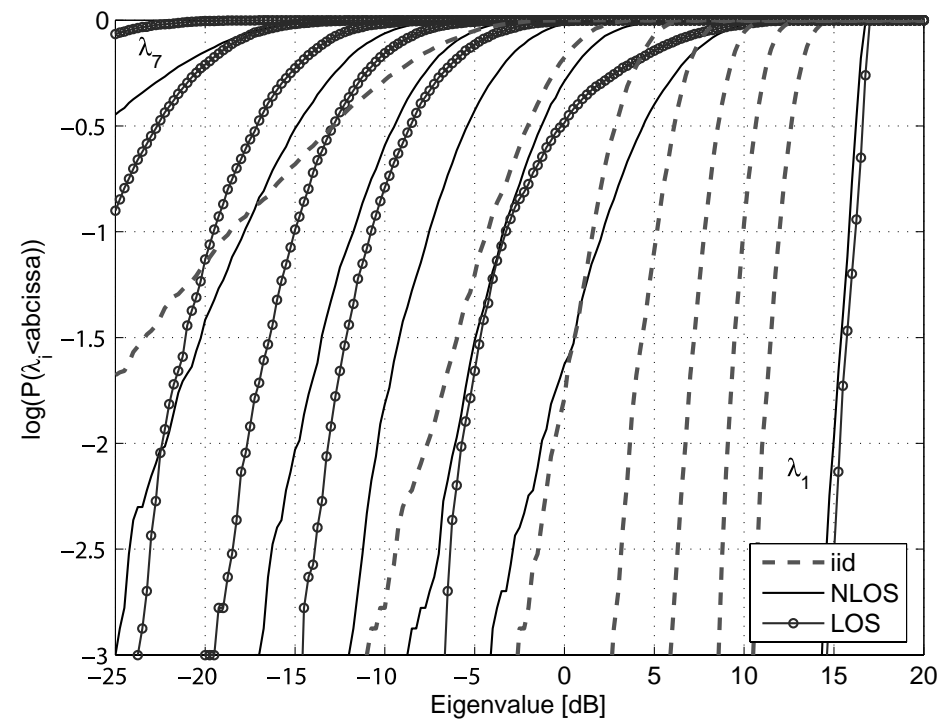

Figure 5. The eigenvalues distribution of the measured channel correlation matrix in NLOS and LOS traveling routes.

$-1 \mathrm{~dB})$. It is worthy to notice that the iid channel maintains seven spatial channels available $90 \%$ of the time. The distribution of each eigenvalue represents the power gain in each spatial channel. It can be seen that in both scenarios the first spatial channel has high power gain while the rest of the eigenvalues have less power gain compared to the first one. For instance, in NLOS traveling route the second and the third eigenvalues are about $13.7 \mathrm{~dB}$ and $19 \mathrm{~dB}$ below the first eigenvalue $90 \%$ of the time, respectively. Having one spatial channel with large power gain and relatively lower power gain spatial channels reflects correlated propagation scenario. In a low correlated propagation environment, as the case of the iid channel, one expect the eigenvalues to have comparable values. It can be concluded that the two propagation environments described by the measurement data exhibit relatively high correlation properties. This could be due to the fact that the propagation takes place through street crossing corners with low angular spread.

The eigenvalues distribution of the channel correlation matrix obtained from the Kronecker model and its smoothed version show useful information about their performance in modeling these propagation scenarios. The KSM between the measured eigenvalues distribution and the eigenvalues distribution obtained from the 


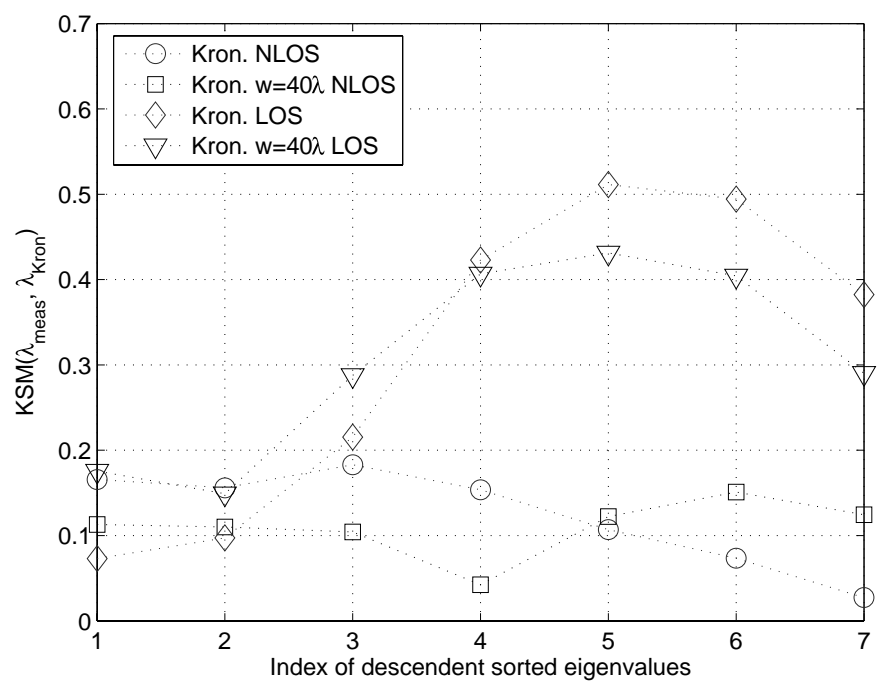

Figure 6. KSM comparing the eigenvalues rendered by the Kronecker model and its smoothed version to the eigenvalues of the measured channel correlation matrix in NLOS and LOS traveling routes.

Kronecker model and its smoothed version with $w=40 \lambda$ are shown in Figure 6. The kronecker model and its smoothed version show different degree of accuracy in rendering the different measured eigenvalues. In NLOS traveling route the spatial smoothing improves the accuracy of rendering the first four large eigenvalues while slightly results in less accurate estimation of the last three eigenvalues. This is due to the fact that the small eigenvalues have very random fluctuations and spatial smoothing will reduce this randomness. On the other hand, in LOS traveling route spatial smoothing with the same window size improves the accuracy of the last four eigenvalues with less accuracy in the first eigenvalues. Generally, one can conclude that both the Kronecker model and its smoothed version fail to render the eigenvalues of the measured channel correlation matrices in both traveling routes since non of the eigenvalues rendered by the Kronecker model and its smoothed version pass the KS test at $95 \%$ significance level.

\subsection{Channel Capacity Analysis}

Under the high data rate signaling scheme and with the equal power allocation strategy, the channel capacity will be the sum of the capacities supported by each spatial channel. Therefore, the total 
channel capacity at each channel realization can be written as:

$$
c=\sum_{i=1}^{R\left(\mathbf{H H}^{H}\right)} \log \left(1+\lambda_{i} \frac{\rho}{N_{t}}\right) \quad \mathrm{b} / \mathrm{s} / \mathrm{Hz}
$$

where $\rho=\frac{\sigma_{x}^{2}}{N_{0}}$ is the average receive signal to noise ratio (SNR).

Figure 7 shows the CDF of the channel capacity obtained from the measurement data and predicted by the Kronecker model and its smoothed version with $w=40 \lambda$ at $20 \mathrm{~dB}$ SNR. The capacity of the iid channel is also shown for sake of comparison. Comparing the capacities from the measured data to the capacity from the iid channel it can be clearly seen that the measured scenarios exhibit correlated paths which degrades the channel capacity relative to the iid capacity significantly. The median of the measured channel capacities are $14.24 \mathrm{~b} / \mathrm{s} / \mathrm{Hz}$ and $20.74 \mathrm{~b} / \mathrm{s} / \mathrm{Hz}$ below the iid channel, for NLOS and LOS traveling routes, respectively.

As it can be seen from the figure that the Kronecker model overestimates and underestimates the channel capacity of the NLOS and LOS traveling routes, respectively. The error in the median capacity in both routes is about $1 \mathrm{~b} / \mathrm{s} / \mathrm{Hz}$. Relative to the median of the measured channel capacity, $24 \mathrm{~b} / \mathrm{s} / \mathrm{Hz}$ in NLOS route and $17.5 \mathrm{~b} / \mathrm{s} / \mathrm{Hz}$ in LOS route, these errors as percentages are about $4.16 \%$ and $5.71 \%$ in NLOS and LOS traveling routes, respectively, which are not very significant. It should be noticed that underestimating the channel capacity is a consequence of overestimating the propagation environment correlation properties and vice versa. The effect of spatial smoothing on the performance of the Kronecker model in predicting the measured channel capacity in NLOS route is clear. Since the accuracy of rendering the first four eigenvalues has been improved by spatial smoothing, the performance of the channel capacity also does. However, in LOS traveling route spatial smoothing did not help to improve the accuracy of the predicted channel capacity. This is again due to the error in rendering the eigenvalues of the measured channel correlation matrix.

\subsection{Symbol Error Rate Analysis}

The SER performance of minimum mean square error (MMSE) receiver with quadrature phase shift keying (QPSK) modulation over the measured and modeled channels was simulated and the results are shown in Figure 8. The SER performance of the iid channel is also shown. One can observe the degradation in the SER performance achieved over the measured channel relative to the iid channel due to 


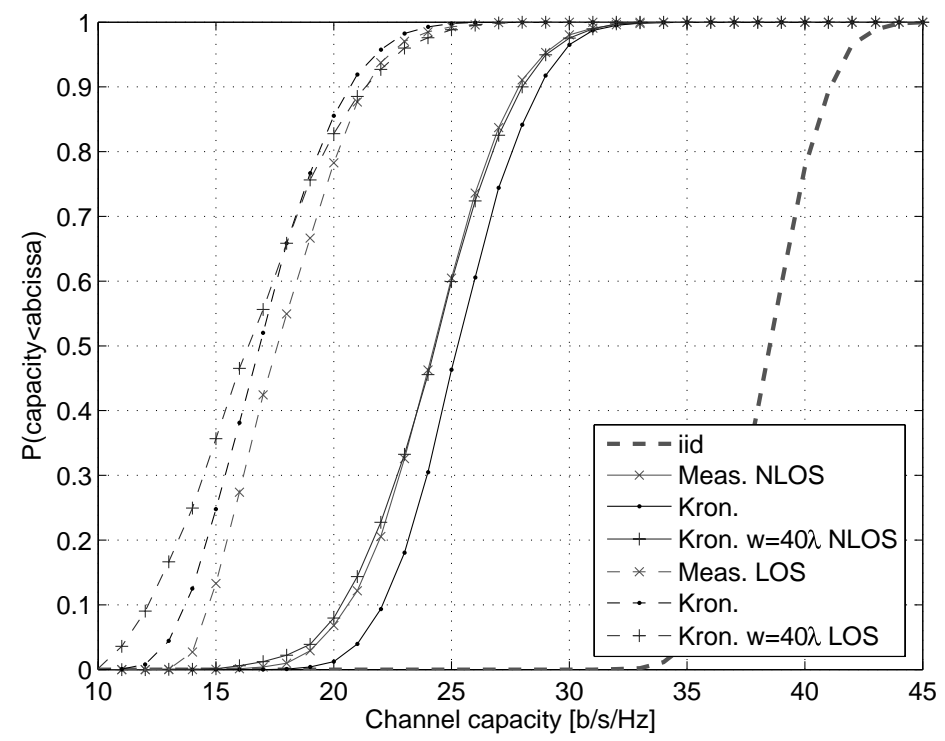

Figure 7. CDF of the achievable channel capacity over the measured and modeled channels at $20 \mathrm{~dB}$ SNR.

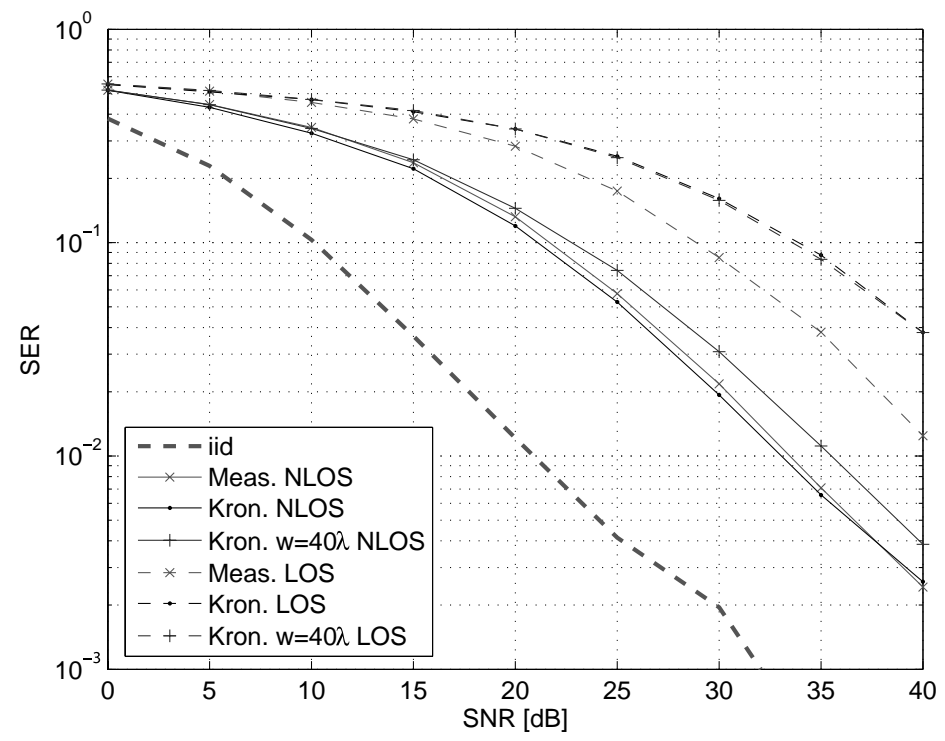

Figure 8. Simulated SER performance of MMSE receiver with QPSK modulation over the measured and modeled channels. 
Table 1. Percentage of model error.

\begin{tabular}{|c|c|c|}
\hline & NLOS & LOS \\
\hline Kronecker & 79.67 & 102.44 \\
Kronecker $w=40 \lambda$ & 56.70 & 99.03 \\
\hline
\end{tabular}

spatial correlation. At low SNR the SER performance based on the measured data and predicted by the Kronecker model and its smoothed version is very similar. This is could be due to the fact that at low SNR the SER performance is dominated by the receiver noise more than the characteristics of the channel matrix. The errors in the modeled channel are not significant at low SNR to affect the SER performance. At SNR higher than $20 \mathrm{~dB}$ the difference between the measured SER performance and the predicted one starts to become significant. For instance, in LOS traveling route at $4 \times 10^{-2}$ SER the mismatch between the measured and predicated SER is about $5 \mathrm{~dB}$ in the required SNR. In NLOS traveling route with high SNR the Kronecker model out performs its smoothed version in terms of accurate SER performance predication.

While the MIMO channel capacity is dominated by the first significant eigenvalues, the SER performance of spatial multiplexing scheme is largely affected by the accuracy of the low eigenvalues of the channel correlation matrix. Spatial smoothing improves the accuracy of the first four large eigenvalues in NLOS route and therefore results in more accurate channel capacity estimation than the Kronecker model. On the other side, the Kronecker model predicates more accurate SER performance in high SNR scenario. This is because the Kronecker model has more accurate estimation of low eigenvalues. The errors in the rendered large eigenvalues will not have noticeable effect on their SER performance but the errors in the rendered small eigenvalues will have significant effect on their SER performance.

\subsection{Model Error Analysis}

The model error $\psi$ measures how well the full channel correlation matrix can be modeled as the Kronecker product of the correlation matrices seen by both ends [3]. The model error is defined as:

$$
\psi=\frac{\left\|\mathbf{R}-\mathbf{R}_{k r o n}\right\|_{F}}{\|\mathbf{R}\|_{F}}
$$

The model error for the Kronecker model and its smoothed version in these propagation scenarios is given in Table 1. Spatial smoothing 
results in reduction of the model error percentage in both propagation scenarios. This is due to the fact that spatial smoothing results in decorrelation of the transmitter and receiver correlation matrices. In NLOS route spatial smoothing reduces the model error percentage about $22.97 \%$ and in LOS route the reduction is only $3.4 \%$. However, the model error remains high and spatial smoothing can not completely decorrelates the two ends correlation matrices. It should be noticed that the model error performance measure is independent on the other performance measures considered above. High model error does not necessary mean high error in the predicted channel capacity and SER performance rather than it means that the Kronecker structure is unappropriate for modeling the full channel correlation matrix. Similar observations were reported in [6]. This could be due to the dependency of the correlation properties at both ends.

\section{CONCLUSIONS}

Based on data measured in outdoor microcellular environment we have investigated the effect of spatial smoothing on the performance of the Kronecker MIMO radio channel model. We have considered different performance measures. It is noticed that the different performance measures require different accuracy of the eigenvalues of the channel correlation matrix. We can conclude that the results predicted with the Kronecker model should be interpreted with care.

\section{REFERENCES}

1. El-Sallabi, H. M. and P. Vainikainen, "Radio wave propagation in perpendicular streets of urban street grid for microcellular communications. Part I: Channel Modeling" Progress In Electromagnetic Research, PIER 40, 229-254, 2003.

2. Kermoal, J. P., L. Schumacher, K. I. Pedersen, P. E. Mogensen, and F. Frederiksen, "A stochastic MIMO radio channel model with experimental validation," IEEE Journal on Selected Areas in Commun., Vol. 20, No. 6, 1211-1226, 2002.

3. Yu, K., M. Bengtsson, B. Ottersten, D. McNamara, P. Karlsson, and M. Beach, "Modeling of wideband MIMO radio channels based on NLOS indoor measurements," IEEE Trans. on Vehicular Technology, Vol. 53, No. 3, 655-665, 2004.

4. Abouda, A. A., H. M. El-Sallabi, and S. G. Häggman, "Effect of antenna array geometry and ULA azimuthal orientation on 
MIMO channel properties in urban city street grid," Progress In Electromagnetic Research, PIER 64, 257-278, 2006.

5. Oz̈celik, H., M. Herdin, W. Weichselberger, J. Wallace, and E. Bonek, "Deficiencies of 'Kronecker' MIMO radio channel model," IEE Electonic Letters, Vol. 39, No. 16, 1209-1210, 2003.

6. McNamara, D. P., M. A. Beach, and P. N. Fletcher, "Spatial correlation in indoor MIMO channels," Proc. of IEEE Indoor and Mobile Radio Communications, Vol. 1, 290-294, 2002.

7. Shan, T. J., M. Wax, and T. Kailath, "On spatial smoothing for direction of arrival estimation of coherent signals," IEEE Trans. Acoust. Speech, Signal Processing, Vol. ASSP-33, No. 4, 806-811, 1985.

8. Pedersen, K. I., P. E. Mogensen, and B. H. Fleury, "Spatial channel characteristics in outdoor environments and their impact on BS antenna system performance," Proc. of IEEE Conference on Vechular Technology, Vol. 2, 719-724, 1998.

9. Boes, D. C., F. A. Graybill, and A. M. Mood, Introduction to the Theory of Statistics, 3rd edition, McGraw-Hill, New York, 1974. 